CORRIGENDUM

doi:10.1038/nature 10420

\title{
Ephrin Bs are essential components of the Reelin pathway to regulate neuronal migration
}

Aycan Sentürk, Sylvia Pfennig, Alexander Weiss, Katja Burk \& Amparo Acker-Palmer

Nature 472, 356-360 (2011)

In this Letter we made errors in representative image choice, including mislabelling of images or choosing an image from the inappropriate genotype. In all cases, choice of images was completely independent of the data analysis and so none of the conclusions in our original Letter are affected. We apologise for any confusion these errors may have caused.

Figure 1a depicts a Tbr1 staining of the adult mouse cortex for four different genotypes. In the process of choosing representative pictures that reflect the results of our analysis shown in Fig. 1b, cropped images from original pictures were inadvertently mislabelled and used incorrectly. We provide below a corrected version of Fig. 1a with new representative images for the following genotypes: WT and Reln ${ }^{+/+}$; $\mathrm{Efnb3}^{-/-}$. A new high-magnification picture for WT is also shown in the two rightmost panels. Original images for every genotype and additional examples are shown in the Supplementary Information of this Corrigendum.

Figure 1c depicts a Brn1 staining of the E17.5 mouse cortex for five different genotypes. In the process of figure assembly cropped images from original pictures were inadvertently mislabelled and used incorrectly. We provide below a corrected Fig. 1c with a new image for $\operatorname{Reln}^{+/+} ; \mathrm{Efnb3}^{-/-}$. In the ephrinB3 compound mice $\left(\mathrm{Reln}^{+/-}\right.$; $\left.\mathrm{Efnb}^{-{ }^{-}}\right)^{\mathrm{Brn} 1^{+}}$cells aberrantly accumulate in the lower layers of the cortex and do not migrate to the upper layers, resembling the Reeler $\left(\operatorname{Reln}^{-/-}\right)$phenotype. Original pictures and additional examples are shown in the Supplementary Information of this Corrigendum, where arrows indicate the distribution of $\mathrm{Brn}^{+}$cells. We have also included results from a new, reproduced experiment recently performed with an additional cohort of animals that shows exactly the same results.

In Fig. 1d, the second panel, labelled ' $\operatorname{Reln}^{+/+} ; E f n b 3^{-/-}$should instead be labelled ' $\mathrm{Reln}^{+/-}$'. In the Methods summary section 'Stimulation of neurons', "Cortical neurons from E14.5 were grown ...." should instead read "Cortical neurons from E15.5 were grown....".

Further errors in the Supplementary Information of the original Letter are described and corrected in the Supplementary Information of this Corrigendum.

Supplementary Information is linked to the online version of the Corrigendum at www.nature.com/nature.

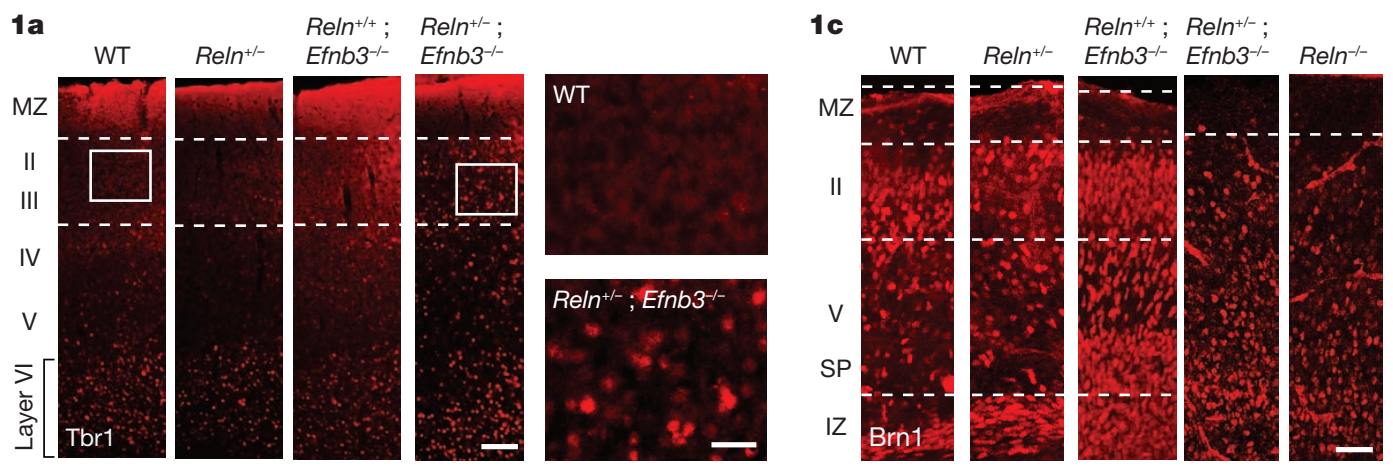

Figure $1 \mid$ Corrected Fig. 1a (with two new panels) and corrected Fig. 1c. 